\title{
PRENATAL ETIOLOGIES OF WEST SYNDROME
}

The etiology of West syndrome (WS) with special reference to prenatal factors was investigated in 180 patients admitted between 1969 and 1990 to the Department of Child Neurology, Okayama University Medical School, Japan. The most common cause was prenatal, accounting for 77 cases (43\%). The frequency of perinatal factors was $14 \%$, and postnatal $7 \%$. Only 18 cases $(10 \%)$ were idiopathic; in 48 the etiology was uncertain. Among prenatal factors, tuberous sclerosis was most frequent, occurring in 23 (30\%) of prenatal and $13 \%$ of the total cases. Chromosome abnormalities were found in 10 (trisomy 21 in 6), and cerebral dysgenesis in 10 . Seizure remission (47\% of cases) and intellectual development ( $\mathrm{IQ}<25$ in $65 \%$ ) were significantly lower in the preratal group compared to idiopathic cases. Pyridoxal phosphate (100-400 mg daily, orally) tried in 170 cases was followed by seizure remission in $12 \%$ of the total, $12 \%$ of prenatal cases, and $28 \%$ of idiopathic cases. ACTH $(0.25 \mathrm{mg} / \mathrm{d} / \mathrm{im}$ for 26 days) was effective in $89 \%$ of 114 cases treated, including 40 (85\%) prenatal cases; seizure remission with ACTH was similar in all etiological groups. (Ohtahara $S$ et al. Prenatal etiologies of West syndrome. Epilepsia July/Aug 1993; 34:716-722). (Reprints: Prof S Ohtahara, Dept of Child Neurology, Okayama University Medical School, Shikatacho, Okayama, Japan).

COMMENT. In this issue of Epilepsia, several papers refer to West syndrome (WS), 4 from the Neuropaediatric Dept, Hopital Saint-VincentDe-Paul, Paris: 1) Cusmai $\mathrm{R}$ et al, reviewing 32 patients with perinatal insults, found a better prognosis in children with periventricular leukomalacia or localized porencephalic cysts than in those with diffuse cerebral lesions or extensive cysts; 2) van Bogaert P, Dulac O et al report that the MRI in 46 patients with WS of unknown etiology was more informative than CT, demonstrating one case of delayed myelination and 4 with focal lesions; 3 ) Jambaque I et al found that visual inattention, an early manifestation of WS, was frequently associated with long-term cognitive defects and parieto-occipital abnormalities in SPECT studies; and 4) Plouin P et al, using 24-hour ambulatory EEG monitoring, recorded partial seizures in 31 patients with an unfavorable outcome.

Additional articles on WS in Epilepsia July/Aug 1993; 34 : include:

5) persisting hypsarrhythmia during a cluster of infantile spasms correlated with a favorable outcome, in a report from the Bambino Gesu Children's Hospital, Rome, Italy (Fusco L, Vigevano F); 6) the term modified hypsarrhythmia should be discarded, and unusual features of interictal EEGs in infantile spasms should be specified, according to Watanabe $\mathrm{K}$ et al, Dept of Pediatrics, Nagoya University, Japan; and 7) infantile spasms, with hypsarrhythmia, retardation and a poor prognosis, occurring in one member of a family with benign familial 
neonatal convulsions, is a unique case report from Tokushima

University, Japan (Mori K, Yano I, Hashimoto T).

\section{EPILEPSY SURGERY AND TUBEROUS SCLEROSIS}

The results of operation for treatment of epilepsy in 5 male and 4 female patients with tuberous sclerosis (TSC) in a 5-year period from 1986-1990 are reported from the Mayo Clinic. Pathological examination of resected lesions demonstrated 7 cortical tubers and 2 astocytic hamartomas. MRIs correlated with clinical and focal EEG findings. Seven patients are free from seizures and 2 are partially controlled. In selected TSC patients with single or multiple cerebral lesions, both the primary and secondary epileptogenic lesions were modified by removal of the primary ictal focus. (Bebin EM, Kelly PJ, Gomez MR. Surgical treatment for epilepsy in cerebral tuberous sclerosis. Epilepsia July/Aug 1993; 34:651-657). (Reprints: Dr MR Gomez, Dept of Neurology, Mayo Clinic, 200 First St, SW, Rochester, MN 55905).

COMMENT. Results of surgery for epilepsy due to cortical dysplasia (CD) are reviewed in 17 patients treated at the Maudsley Hospital, London (Hirabayashi S et al. I Neurol Neurosurg Psychiatry July 1993; $\underline{56}$ :765). Only 6 became free or almost free from seizures, and 8 had no relief. Outcome related to the extent of the pathology but not to histology. Lesions outside the temporal and frontal lobes and generalized interictal EEG abnormalities, reflecting extensive or multiple lesions, correlated with poor surgical outcome. MRI was abnormal in 5 of 7 patients examined, contrasting with a low detection rate of abnormalities by CT scan. The use of MRI and PET should improve the identification of CD patients amenable to surgery.

\section{SEIZURES AND NEUROFIBROMATOSIS I}

Twenty-two (6\%) patients, ages 5 to 57 years, had developed seizures among 359 attending a neurofibromatosis clinic at the Dept of Neurology, Children's Hospital, Harvard Med School, Boston. The majority of seizures could be attributed to causes unrelated to NF1: 6 had febrile seizures and 3 had primary generalized epilepsy with onset before 5 years. Complex partial seizures in $9(41 \%)$, infantile spasms in 1, and seizures with aqueductal stenosis in 2 may have been caused by NF1, but no specific brain lesions were detected. None of the seizures was a symptom of brain tumor, and neuroimaging failed to uncover a seizure focus. Routine EEG of all patients with NF1 was considered unproductive. (Korf BR, Carrazana E, Holmes GL. Patterns of seizures observed in association with neurofibromatosis 1. Epilepsia July/Aug 1993;34:616-620). (Reprints: Dr BR Korf, Children's Hospital, 300 Longwood Ave, Boston, MA 02115). 